\title{
PHOTON-NEUTRINO INTERACTIONS
}

\author{
G.Karl and V.Novikov*, \\ Department of Physics, University of Guelph, \\ Ontario, Canada N1G 2W1 \\ and Guelph-Waterloo Physics Institute
}

\begin{abstract}
We discuss the interaction of photons with neutrinos including two lepton loops. The parity violation in the $\gamma \nu \rightarrow \gamma \nu$ channel due to two lepton loops is substantially enhanced relative to the one lepton loop contribution. However there is no corresponding enhancement in the parity conserving amplitude in either the direct or the cross channel $\gamma \gamma \rightarrow \nu \bar{\nu}$
\end{abstract}

\section{Introduction and Summary}

The photon-neutrino interaction is very feeble, involving only electrically neutral external particles. The cross sections are exceedingly tiny. Therefore this interaction can only be of astrophysical interest. Chiu and Morrison [1] proposed long ago that photon conversion to neutrinos may play a role in supernova cooling. Another possible application involves the propagation of light waves through a handed neutrino sea [2] which results in optical activity (birefringence). There may perhaps be effects in the cooling of the early universe. For all these reasons there has been some small theoretical interest

${ }^{*}$ On leave of absence from the Institute of Theoretical and Experimental Physics, Moscow, Russia 
in this interaction [3]. We consider here the interaction at energies which are small compared to the mass of the weak bosons. At these energies the weak interaction can be described by a Fermi type effective theory. To have $\nu \gamma$ interaction one needs virtual leptons which couple both weakly and electromagnetically, thus lepton loops in Feynman graphs. Contrary to intuition a two loop graph dominates the parity violation. In this section we summarize our results using only dimensional arguments. A more technical description is in the next sections.

For supernova cooling the amplitude for the annihilation $\gamma \gamma \rightarrow \nu \bar{\nu}$ is relevant, while for photon propagation in a neutrino sea the parity violation in the cross(scattering) channel $\gamma \nu \rightarrow \gamma \nu$ is important. We discuss first the annihilation process where the two loop contribution turns out to be small. It is natural to expect higher order graphs to be smaller but as mentioned we find a violation of this rule here. This is reminiscent of a surprise in coherent photon scattering from atoms discussed by G.E.Brown and Woodward and later Peierls [4.

The earliest estimate for the annihilation amplitude used the Fermi theory of weak interactions [1], but Gell-Mann [5] noted that in $V-A$ theory this amplitude vanishes for a point interaction of four fermions. This can be understood physically without any detailed computation. In Fermi Theory the annihilation amplitude is described by a triangle graph, with an electron running in the triangular loop (see Fig.1).

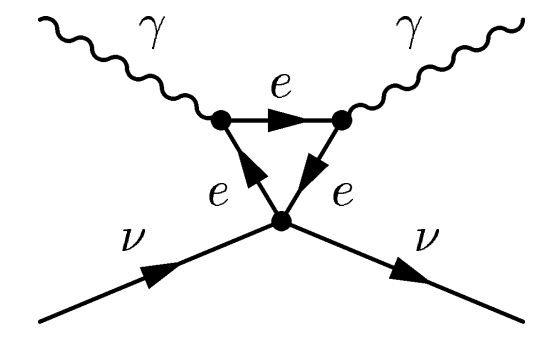

Figure 1: $\gamma \nu$ scattering in the four-fermion Model.

Two of the vertices of the triangle are electromagnetic (where the two photons couple) and the third vertex is weak, where the two neutrinos emerge. The amplitude has magnitude $G_{F} \alpha(p k)$, where $p$ and $k$ are the four-momenta of the neutrino and photon, and $p k$ is the only non-vanishing relativistic invariant for the process (the Feynman amplitude is dimensionless which 
brings the factor $p k$ ). Because the two neutrinos emerge from the same spacetime point they can have no relative orbital angular momentum (s-wave) . With vector or axial vector coupling $(V-A)$, the spins of the fermion-antifermion pair must be parallel. A theorem of Landau and Yang [6] forbids two free photons in a state with total angular momentum one. So the process is forbidden at order $G_{F}$ in $V-A$ theory. Gell-Mann also noted that this proof does not hold if there is a weak boson mediating the weak interaction, so that the two neutrinos are emitted at separate space-time points.

With a weak boson to mediate the interaction, the (parity conserving) amplitude was estimated by Levine [7, and his estimate remains valid in the standard model, as noted by a number of authors [3, 8. With the weak boson the loop changes from a triangle to a square, with the weak boson providing one of the sides (see Fig.2).

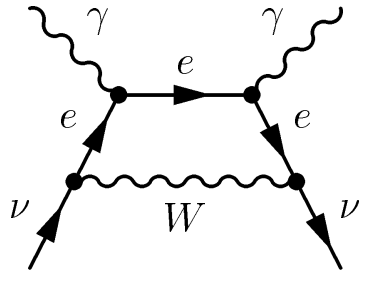

a)

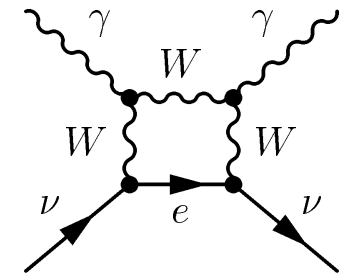

b)

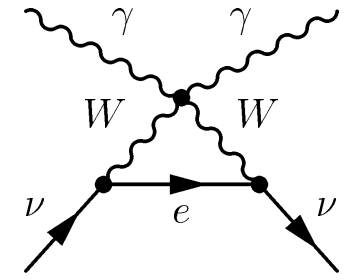

c)

Figure 2: $\gamma \nu$ scattering in the Standard Model.

This gives an additional factor of $\left(p k / M_{W}^{2}\right)$ to first order in the momenta $p$ and $k$. This suppression factor vanishes when the boson mass goes to infinity, in agreement with Gell-Mann's theorem. The precise cross section for the annihilation was computed by Dicus and Repko [8] to order $\left(G_{F} \alpha\right)^{2}$ :

$$
d \sigma / d z=\left[\left(G_{F} \alpha\right)^{2} / 32 \pi^{3}\right]\left[\omega^{6} / M_{W}^{4}\right]\left[1-z^{4}\right],
$$

where $z$ is $\cos \theta$ and $\omega$ the photon energy(in CoM system: $p k=\omega^{2}(1-z)$ ) and we have dropped logarithms. This is in agreement with the one loop Feynman amplitude described above, up to a constant factor:

$$
T^{(1)}=\left[G_{F} \alpha \omega^{4} / M_{W}^{2}\right][\text { angularfunction }][\text { logarithms }] .
$$

The suppression factor $\left(\omega^{2} / M_{W}^{2}\right)$ may be interpreted as a $(k R)^{2}$ factor associated with a d-wave for the two neutrinos. Therefore the leading order 
amplitude may be thought of as anomalously small compared to initial expectations [8] .

When we go to the next order in weak interactions there is a set of diagrams in which the two neutrinos are emitted at a separation of the order of the Compton wavelength of the electron instead of the Compton wavelength of the weak boson. The diagram remains a square with two adjacent weak (order $G_{F}$ ) vertices, separated by an electron-neutrino loop. The two final neutrinos are emitted at the two weak vertices, and the two photons couple at the two remaining vertices (see Fig.3).

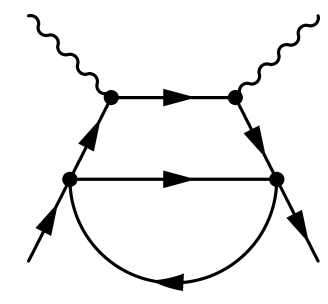

Figure 3: The sample of two-loop contribution into $\gamma \nu$ scattering

We can estimate the Feynman amplitude associated with the two-loop graph in lowest order using the same dimensional arguments as in the case of the one-loop result, changing the $M_{W}$ with $m_{e}$ in the denominator:

$$
T^{(2)}=\left[G_{F}^{2} \alpha(p k)^{3} / m_{e}^{2}\right]=\left[G_{F}^{2} \alpha \omega^{6} / m_{e}^{2}\right][\text { angular function }]
$$

and therefore the ratio of the two-loop amplitude to the one loop is:

$$
T^{(2)} / T^{(1)}=G_{F} \omega^{2}\left(M_{W}^{2} / m_{e}^{2}\right) .
$$

The bracketed ratio of the two squared masses is very large $\sim 10^{10}$ so that the cross-section is dominated by the leading term $T^{(1)}$ only at low energies $\left(\omega<m_{e}\right)$. However at intermediate photon energies $\omega>m_{e}$ our estimate of the two loop contribution is no longer valid since the ratio $(p k) / m_{e}^{2}$ is no longer small. To estimate what happens at these energies we take the limit where this ratio is large and then we expect $m_{e}^{2}$ to be replaced by $(p k)$. Then the ratio of the two loop contribution to the one loop contribution becomes of order $G_{F} M_{W}^{2}$ which is a small number (of order $10^{-2}$ ) independent of energy. Therefore there is no enhancement of the annihilation amplitude due to two loop diagrams. This is in accord with expectations. 
We come now to the parity violating amplitude in the cross channel. This was first studied by Royer [2], using the triangle graph, and his result criticized by Stodolsky [2] on the basis of Gell-Mann's theorem. More recently estimates have been proposed using the standard model 3 and the one lepton loop graph, for both real and virtual photons. Here we show that these one loop estimates are all negligible when compared to the two lepton loop estimate-for real photons.

In the cross channel the parity violating amplitude has to be odd under inversion of the coordinates. The precise form is discussed in section 2, and given below. As a result, parity violation appears dimensionally as a two loop effect even for a one loop amplitude. So it is natural to consider two loop terms together with one loop terms.

The estimate of the parity violating Feynman amplitude in the standard model can be written in the form [3]:

$$
T_{p v}^{(1)}=C \frac{G_{F} \alpha(p k)^{2}}{\sqrt{2} M_{W}^{2}} \epsilon_{\mu \nu \alpha \beta} \epsilon_{\mu}(k) \epsilon_{\nu}^{*}(k)\left(\frac{p_{\alpha} k_{\beta}}{M_{W}^{2}}\right) \sim 194 G_{F} \alpha \omega^{2}\left(\frac{\omega^{4}}{M_{W}^{4}}\right),
$$

where $C$ is a constant involving logarithms [3] and in the second line we evaluate the magnitude of the first expression in the forward direction for photons of energy $\omega$ where $p k=2 \omega^{2}$. This formula should apply for $\omega<M_{W}$.

With two lepton loops the corresponding parity violating Feynman amplitude has the form(for $\omega<m_{e}$ )

$$
T_{p v}^{(2)}=\frac{3 G_{F}^{2} \alpha(p k)^{2}}{32 \pi^{3}} \epsilon_{\mu \nu \alpha \beta} \epsilon_{\mu}(k) \epsilon_{\nu}^{*}(k)\left[\frac{p_{\alpha} k_{\beta}}{m_{e}^{2}}\right] \sim 0.025 G_{F}^{2} \alpha \omega^{6} / m_{e}^{2} .
$$

If we compare the two lepton loop to the one lepton loop result we find

$$
T_{p v}^{(2)} / T_{p v}^{(1)} \sim\left(10^{-4}\right) G_{F} M_{W}^{2}\left[M_{W}^{2} / m_{e}^{2}\right] .
$$

This ratio is quite large and independent of the photon energy below $m_{e} \sim 500 \mathrm{keV}$; its value is approximately $2 \cdot 10^{5}$. Physically this reflects the fact that the two final neutrinos are separated in the two loop Feynman graph by an intermediate electron and a neutrino, and hence a distance of an electron Compton wavelength. Even for photon energies above $1 \mathrm{MeV}$ the corresponding graph with a muon loop enhances the amplitude for photonmuon-neutrino scattering by a factor of 5 . Should such an effect be observed it would indicate a violation of $C P T$ and Lorentz invariance [9. Attempts 
to observe such an effect have been unsuccessful [10]. Why is the situation different with the parity violation in the forward amplitude in the scattering channel? The answer is simple. This amplitude must be odd under parity and therefore be at least cubic in $k p$. So we need an extra factor of $k p$ and this comes (in the one-loop case) with its own denominator $M_{W}^{2}$, for dimensional reasons. Therefore the parity violating amplitude even in the one loop diagram is formally of order $G_{F}^{2}$ and therefore on par with the two loop diagram. However it should be emphasized that even with this large enhancement factor the effect remains extremely small.

For real photons $k^{2}=0$, the two loop estimates are as explained above, dominant. Therefore estimates of the rotatory power of the neutrino sea in the literature [3] have to be multiplied by a factor of $2 \cdot 10^{5}$ to make them right. In particular, using the Equation (31) of Abbasabadi and Repko [3], and applying this correction we get for the rotatory power $\phi / L$ the formula:

$$
\phi / L=0.015 G_{F}^{2} \alpha \omega^{2} T_{\nu}^{2}\left(N_{\nu}-N_{\bar{\nu}}\right) / m_{e}^{2},
$$

which should only apply in the range discussed by Abbasabadi and Repko [3] ( where $T_{\nu}$ is the neutrino Fermi energy).

For off-mass-shell photons the situation is different, since the LandauYang theorem does not apply to off-mass shell photons. Therefore even the lowest triangle graph is viable as was realized by Nieves et al [3]. At low photon energy their one loop estimate remains dominant while at higher photon energies the two loop estimate dominates. The transition between Low and High photon energies $\omega^{*}$, is given by the equation $\omega^{*} E_{\nu}>30 \mathrm{eV}^{2}$, where $E_{\nu}$ is the neutrino energy in the rest frame of the sea. For a standard sea of energy of a few degrees Kelvin the transition occurs in the $\omega \sim 100 \mathrm{KeV}$ region, but for an unconventional sea of Fermi Energy $100 \mathrm{eV}$ [1] the two loop estimate will dominate even for visible photons. For radio waves the one loop estimate dominates for virtual photons.

\section{Effective Lagrangian}

For a systematic study of neutrino-photon interaction it is useful to work with effective Lagrangians.

The simplest example of the Effective Lagrangian is the four-fermion interactions of neutrinos $\nu$ with electrons $e$. For fermions with momenta much 
smaller than intermediate bosons mass one can integrate degrees of freedom associated with $W$ and $Z$ and write the Effective Lagrangian only for fermionic degrees of freedom:

$$
L_{e f f}=\frac{G_{F}}{\sqrt{2}}\left(\bar{\nu} \gamma_{\alpha} \nu\right)\left(\bar{e} \Gamma_{\alpha} e\right)
$$

where $\Gamma_{\alpha}=g_{V} \gamma_{\alpha}+g_{A} \gamma_{\alpha} \gamma_{5}$. In the Standard Model $(S M)$ one finds $g_{V}=$ $\frac{3}{2}-2 \sin ^{2} \theta_{W}, g_{A}=\frac{3}{2}$. For small momenta this effective Lagrangian is as good as the fundamental Lagrangian of the $S M$.

Consider now the process $\nu(p)+\gamma(k) \rightarrow \nu(p)+\gamma(k)$ at momenta smaller than intermediate bosons mass or even smaller than the mass of electron $m_{e}$. Interaction between neutrino and photons occurs only through the interaction of photons with charged virtual particles in loops. Loop diagrams are numerous and a bit complicated. For small momenta $\left(p k / m_{W}^{2}\right) \ll 1$ one can expand $\nu(p)+\gamma(k) \rightarrow \nu(p)+\gamma(k)$ amplitudes in the power series in this small parameter. The lowest terms of this expansion can be represented as a matrix element of the appropriate operators i.e. of the particular terms of the Effective Lagrangian.

Each term of the Effective Lagrangian has to be Lorenz-invariant combination of gauge-invariant electromagnetic field tensor $F_{\mu \nu}$ and left-handed neutrino field $\nu_{L}=\frac{1}{2}\left(1+\gamma_{5}\right) \nu$ and their derivatives. Effective Lagrangian has dimension four: $[L] \sim[m]^{4}$. The operators may have higher dimensions $D$. To preserve correct dimension the coefficients in front of these operators should be proportional to appropriate power of $(1 / m)$, where $m$ is the scale of mass walking inside the loops. The actual calculation of the diagrams gives numerical coefficient in front of the operator.

This line of reasoning is very similar to the naive dimensional arguments given in the Introduction. The only advantage of effective Lagrangian is that within that more advanced approach we get more clear understanding of the structure of the operators (i.e. of the scattering amplitudes).

\section{$2.1 \quad$ P-even scattering amplitude}

\subsubsection{One-loop approximation}

Consider effective Lagrangian for $P$-even $\nu \gamma$-scattering. The photon's part of amplitude should be even under parity, i.e. be the same for right-handed photons and left-handed photons. One has to construct appropriate Lorenz 
invariant operators from the fields $F_{\mu \nu}$ and $\nu_{L}$. The combination of the fields that satisfies all these conditions looks as follows:

$$
L_{e f f} \sim \frac{e^{4}}{m^{4}}\left[F_{\mu \alpha} F_{\mu \beta}\right] \bar{\nu} \gamma_{\alpha} \partial_{\beta}\left(1+\gamma_{5}\right) \nu+\text { h.c. },
$$

It has dimension $D=8$. Matrix element of $L_{e f f}$ for forward scattering gives the amplitude

$$
T \sim \frac{e^{4}}{m^{4}}(p k)^{2} \epsilon(k)^{*} \epsilon(k)
$$

If we identify parameter $m$ in eq.(11) with the largest mass in diagrams (i.e. with $m_{W}$ ) we reproduce well known result [7] up to the numerical constant.

From this exercise it is absolutely clear that non-zero $\gamma \nu$ scattering appears only in the second order in photon momenta, i.e. in order $(p k)^{2}$. Thus one immediately concludes that any results of zero order in $k$, i.e. of order $\left(G_{F} \alpha\right) \sim \alpha^{2} / m_{W}^{2}$ (e.g. such as in ref. [1, 2]), are erroneous.

There is no way to violate Gell-Mann theorem within effective Lagrangians. To get the amplitude of the order $G_{F} \alpha$ one needs operator with $D=6$. By direct inspection one finds that there is no gauge invariant operator with $D=6$.

\subsubsection{Two-loop approximation}

Consider now two-loop amplitudes with light particles (i.e. electrons and neutrino) in intermediate state between two external neutrino vertices. We expect that these diagrams are proportional to $G_{F}^{2} \alpha / m_{e}^{2}$. Thus to preserve correct dimension in effective Lagrangian we need operator of dimension $D=10$. Appropriate effective Lagrangian is

$$
L_{e f f} \sim \frac{G_{F}^{2} \alpha}{m_{e}^{2}}\left[F_{\mu \alpha}\left(\partial_{\gamma} F_{\mu \beta}\right)\right]\left[\bar{\nu} \gamma_{\alpha} \partial_{\beta} \partial_{\gamma}\left(1+\gamma_{5}\right) \nu\right]+\text { h.c. }
$$

For this $L_{e f f}$ the scattering amplitude is of the third order in $(p k)$

$$
T \sim C \frac{G_{F}^{2} \alpha}{m_{e}^{2}}(p k)^{3} \epsilon(k) \epsilon^{*}(k) .
$$

Thus for P-even scattering second order loops give correction of the order $\left(G_{F} M_{W}^{2}\right)\left(p k / m_{e}^{2}\right)$, i.e. small correction to the one-loop result. 


\subsection{Optical activity. P-odd scattering amplitude}

Now let us come back to $P$-odd effects in $\nu \gamma$ scattering and find appropriate operators in $L_{\text {eff }}$ responsible for optical activity. The Lagrangian of dimension $D=8$ that depends on $P$ - odd combinations of photon polarizations has the form

$$
L_{e f f} \sim \frac{1}{m_{W}^{4}}\left[F_{\mu \alpha} \tilde{F}_{\mu \beta}\right]\left[\bar{\nu} \gamma_{\alpha} \partial_{\beta}\left(1+\gamma_{5}\right) \nu\right]+\text { h.c. },
$$

where $\tilde{F}_{\mu \nu}=\frac{1}{2} \epsilon_{\mu \nu \alpha \beta} F_{\alpha \beta}$.

The surprise is that this operator does not work in our case. On can check that the matrix element of $F_{\mu \alpha} \tilde{F}_{\mu \beta}$ between photons with the same momenta and polarization ( forward scattering) is identically zero and this operator of $D=8$ does not contribute into $P$-odd forward scattering. Thus P-odd effects are zero in $(p k)^{2} / m_{W}^{4}$ order. The first nonzero effect is of the third order $\sim(p k)^{3}$. Birefringence of neutrino sea is strongly suppressed!

To find effect in the next order we have to look for the operators of higher dimension $D=10$. One of these operators looks like follows

$$
L_{e f f} \sim \frac{1}{m^{6}}\left[F_{\mu \alpha}\left(\partial_{\gamma} \tilde{F}_{\mu \beta}\right)\right]\left[\bar{\nu} \gamma_{\alpha} \partial_{\beta} \partial_{\gamma}\left(1+\gamma_{5}\right) \nu\right]+h . c .
$$

With this $L_{\text {eff }}$ the forward scattering amplitude for a photon with momentum $k$ and for a neutrino with momentum $p$ is equal to

$$
T=C\left(e^{4} / 8 \pi^{2}\right)\left(p k / m^{2}\right)^{2} \epsilon_{\mu \nu \alpha \beta} \epsilon_{\mu}(k) \epsilon_{\nu}^{*}(k)\left(p_{\alpha} k_{\beta} / m_{W}^{2}\right) .
$$

This amplitude has different contribution to left-handed and to right-handed photons scattering : $T_{L L}=-T_{R R}$.

\section{Actual calculations}

\subsection{One-loop calculations. Real photons}

The actual calculation of the coefficient $\mathrm{C}$ has been done in one loop-approximation in [3] with the results

$$
\left.T=C\left(e^{4} / 8 \pi^{2} s^{2}\right)\left(p k / m_{W}^{2}\right)^{2} \epsilon_{\mu \nu \alpha \beta} \epsilon_{\mu}^{(} k\right) \epsilon_{\nu}^{*}(k)\left(p_{\alpha} k_{\beta} / m_{W}^{2}\right),
$$

where

$$
C=4 / 3\left(\ln \left(m_{W}^{2} / m^{2}\right)-11 / 3\right)
$$


in the third reference in [3] and

$$
C=4 / 3\left(\ln \left(m_{W}^{2} / m^{2}\right)-8 / 3\right)
$$

in the fourth one 3]. The reason for that discrepancy is unknown. Though numerically eqs.(18) and (19) differ by only a few per cent it would be interesting to understand whether there is a correct one-loop calculation.

On the other hand we find that for a $P$-odd effect two-loop diagrams by many order of magnitude larger than one-loop contribution. Thus we can neglect any one-loop results.

\subsection{One-loop calculations. Off-shell photons}

The optical activity for off-shell photon was first considered by Mohanty, Nieves and Pal in [3. They noticed that Gell-Mann prohibition theorem does not work for the off-shell photons. Thus one can expect that off-shell amplitude is of the first order in $1 / m_{W}^{2}$. Indeed

$$
\left.T=\left(e^{4} / 8 \pi^{2} s^{2}\right) \epsilon_{\mu \nu \alpha \beta} \epsilon_{\mu}^{(} k\right) \epsilon_{\nu}^{*}(k)\left(p_{\alpha} k_{\beta} / m_{W}^{2}\right)\left(k^{2} / 6 m_{e}^{2}\right),
$$

where $s=\sin \theta_{W}$.

Eq. (20) differs from the original result of ref. 33 by factor $1 / 2$. The reason is that triangle diagram was missing there. (This is the triangle diagram with $Z$ boson and two photons. For real photons, $k^{2}=0$ the triangle diagrams cancel each other in the SM since the SM is anomaly free. But for off-shell photons each triangle diagram gives a contribution proportional to $k^{2} / m^{2}$. The sum of all triangles is nonzero and the main contribution comes from the electron loop. This contribution has to be taken into account)

\subsection{Two-loop calculation}

The physical reason for the dominance of the two-loop diagrams under the one-loop in P-odd amplitudes is simple. To escape Gell-Mann's restriction one needs non-local interactions in order to include higher orbital momenta of the pair $\nu \bar{\nu}$ into annihilation process. In the one loop approximation the expectation value of the orbital momentum of the neutrino pair is $\sim$ $p / m_{W}$. The factor $1 / m_{W}$ measures the shortest separation of two neutrino during interaction (non-locality). 
In the two-loop approximation the expectation value of neutrino pair orbital moment is $\sim p / m_{e}$. The factor $1 / m_{e}$ is due to $\left(e^{-} e^{+} \nu\right)$ in the intermediate states.

Kinematically $\mathrm{P}$-odd amplitudes are of the order of $(p k)^{3}$ (see section 2.2). On dimensional ground we conclude that two-loop amplitudes are of the same order $(p k)^{3}$ (see secton 2.1.2). Thus one-loop P-odd amplitude has the same dependence on $(p k)$ as the two-loop P-odd amplitude. Moving to the next order in electro-weak interaction we loose a small factor $\alpha / 2 \pi$, but win a great factor $\left(m_{W}^{2} / m_{e}^{2}\right)$. The net effect is

$$
\left(T^{(2)} / T^{(1)}\right) \sim(\alpha / 2 \pi)\left(m_{W}^{2} / m_{e}^{2}\right) \sim 10^{7} .
$$

Actual calculation is rather lengthy. The result is

$$
T^{(2)}=(13 / 27)\left(G^{2} e^{2} / 64 \pi^{4}\right)\left(g_{V}^{2}+g_{A}^{2}\right)(p k)^{2} \epsilon_{\mu \nu \alpha \beta} \epsilon_{\mu}(k) \epsilon_{\nu}^{*}(k)\left(p_{\alpha} k_{\beta} / m_{e}^{2}\right) .
$$

Thus the enhancement factor is

$$
T^{(2)} / T^{(1)}=\left(\alpha / 64 \pi \sin ^{2} \theta_{W}\right)\left(m_{W}^{2} / m_{e}^{2}\right)(13 / 27 C)\left(g_{V}^{2}+g_{A}^{2}\right) \sim 10^{5},
$$

where $C$ is one-loop coefficient from eq.(8). We have lost two order of magnitude compared with naive estimate in eq.(10) mainly due to the large logarithmic coefficient $C$ in one-loop amplitude. Still enhancement factor is very large $\sim 10^{5}$ !

This research was supported by NSERC-Canada and by RFBR grants 00-15-96562.

\section{References}

[1] H.Y.Chiu and P.Morrison, Phys. Rev. Lett. 5 (1960) 573; S.G.Matinyan and N.N. Tsilosuni, Sov.Phys. JEPT 14 (1961) 1195.

[2] J.Royer, Phys. Rev. 174 (1968) 1719; L.Stodolsky, quoted in G.Karl, Canadian Journal of Physics 54 (1976) 568.

[3] see e.g.: S.Mohanty, J.F.Nieves and P.Pal, Phys. Rev. D58 (1998) 093007; Ali Abbasabadi and Wayne W.Repko, Phys.Rev. 
D64(2001)113007; ibid, D67 (2003) 073018; G.Karl and V.A.Novikov, hep-ph/0009012 V.B.Bezerra et al, Phys.Rev D67 (2003) 084011; V.B.Bezerra, C.N.Fereira,J.A.Helayel-Neto, hep-th /0405181.

[4] R.E.Peierls: "Surprises in Theoretical Physics" Princeton University Press,Princeton, NJ 1975

[5] M.Gell-Mann, Phys. Rev. Lett. 6 (1961) 70.

[6] L.D.Landau, Dokl. Akad. Nauk USSR 60, 207 (1948); C.N.Yang, Phys. Rev. 77, 242 (1950)

[7] M.J.Levine, Nuovo Cimento A48 (1967) 67.

[8] Duane A. Dicus and Wayne W.Repko, Phys.Rev. D48(1993) 5106

[9] S.Coleman and S.Glashow, hep-ph/9812418 R.Jackiw and V.A.Kostelecky, Phys. Rev. Lett. 82 (1999) 3572

[10] see e.g. J.N.Clarke, G.Karl and P.J.S.Watson, Canadian Journal of Physics 60 (1982) 1561.

[11] V.M.Lobashev et al, Phys. Lett. B460 (1999) 227. 